\title{
Metodologias usadas no desenvolvimento de Jogos Digitais Educacionais: uma revisão da literatura
}

\author{
Paulo Henrique de Souza Oliveira ${ }^{1}$, Lisandra Manzoni Fontoura ${ }^{1}$, Roseclea Duarte \\ Medina $^{1}$
}
${ }^{1}$ Programa de Pós-Graduação em Ciência da Computação - Universidade Federal de Santa Maria (UFSM)
paulo.oliveiraeiffarroupilha.edu.br.edu.br, lisandra.fontoura@ufsm.br, rosedinf.ufsm.br

\begin{abstract}
The development of educational electronic games has shown to be a powerful tool for teaching and learning. Using an appropriate development methodology provides to achieve the pedagogical objectives, developing a game that meets the requirements initially defined and presents the expected quality. There is still no consensus in the literature regarding which methodologies should be used for the development of pedagogical electronic games. This article describes a systematic literature review that aims to identify the methodologies that have been used in the development of educational electronic games. In the analysis, carried out from the analysis of 19 selected articles, we concluded that although there is no methodological consensus, nor much concern to adaptation to specific cases, user participation, use of agile techniques and potential for the development of computational thinking
\end{abstract}

Resumo. $O$ desenvolvimento de jogos eletrônicos com propósito pedagógico tem se mostrado uma poderosa ferramenta para o ensino-aprendizagem. Utilizar uma metodologia de desenvolvimento adequada proporciona atingir os objetivos pedagógicos, desenvolvendo um jogo que satisfaça os requisitos inicialmente definidos e apresente a qualidade esperada. Não existe, ainda, um consenso na literatura, em relação a quais metodologias devem ser utilizadas para o desenvolvimento de jogos eletrônicos pedagógicos. Este artigo descreve uma revisão sistemática de literatura que tem como objetivo identificar as metodologias que vêm sendo utilizadas no desenvolvimento de jogos eletrônicos educacionais. Na análise, realizada a partir da análise de 19 artigos selecionados, constatou-se que apesar de não haver um consenso, existem similaridades nos processos, principalmente no que se refere à adaptação para casos específicos, participação dos usuários, uso de técnicas ágeis e potencial para o desenvolvimento do pensamento computacional.

\section{Introdução}

O uso de jogos como recurso pedagógico vem sendo explorado há vários anos. Isso se deve ao grande potencial que esse recurso vem demonstrando como facilitador do processo de ensino-aprendizagem, incluindo fatores como fluxo, imersão (sensorial e 
imaginativa), competência, desafio, afeto e tensão nas possibilidades de interação com o estudante. É um tipo atrativo de mídia, principalmente pela imersão e interatividade que proporciona aos usuários" [PERUCIA, 2007]. Sabe-se que "jogos digitais são aplicações que possuem, na maioria das vezes, um processo de desenvolvimento específico" [PERUCIA, 2007], devido à diversidade de técnicas disponíveis e sua adequação restrita a alguns tipos de aplicação.

Além disso, "em projetos de pequeno porte não há necessidade de seguir todo o ciclo de desenvolvimento, pois somente o Game Design Document (GDD), documento gerado durante as etapas de Game Design e Design Document é o bastante para que o projeto seja executado de forma satisfatória" [PERUCIA, 2007]. Assim, cada desenvolvedor pode fazer uso de técnicas existentes, criar sua própria técnica ou ainda mesclar metodologias existentes para adequar a sua necessidade específica.

Nesse contexto, esse artigo se propõe a revisar a literatura científica para identificar as metodologias utilizadas no desenvolvimento de jogos eletrônicos educacionais, no contexto acadêmico, e os requisitos metodológicos específicos desse tipo de processo, com ênfase na forma de documentação utilizada e, dessa forma responder as seguintes questões de pesquisa: 1) Quais metodologias são utilizadas no desenvolvimento de jogos eletrônicos educacionais no contexto acadêmico?; 2) Quais os requisitos metodológicos específicos no desenvolvimento de jogos educativos?; 3) Como é feita a documentação (Game Design Document)? As respostas a essas questões visam elencar as ferramentas, técnicas ou mesmo processos (padronizados ou não) que representem boas práticas no desenvolvimento dessa categoria de software, afim de instrumentalizar projetos futuros.

No desenvolvimento desse estudo, foram identificados outros trabalhos realizados com propósito semelhante. Eles são relativamente antigos, considerando a rápida evolução dessa temática e não se preocupam com o levantamento de critérios comuns usados no desenvolvimento de jogos desse tipo.

Este artigo está organizado da seguinte forma: na Seção 2 é descrito o protocolo utilizado na revisão sistemática; na Seção 3 são apresentados os dados extraídos e sua sintetização na formulação de respostas para as questões de pesquisa; na Seção 4, são apresentadas as considerações finais e possibilidades de trabalhos.

\section{Protocolo da Revisão Sistemática}

A fim de estabelecer os critérios comumente referenciados na definição de metodologias para o desenvolvimento de jogos educacionais, realizou-se uma revisão sistemática da literatura (RSL) seguindo o roteiro proposto por [KITCHENHAM, 2004] que define esse tipo de estudo como "um meio de identificar, avaliar e interpretar toda a pesquisa disponível relevante para uma questão de pesquisa específica, tópico de área ou fenômeno de interesse". Para isso, a autora estabelece um roteiro organizado em três etapas fundamentais, sendo elas o planejamento, a condução e o relato.

No "planejamento", objetiva-se constatar a necessidade da realização do estudo e criar o protocolo que será seguido durante o processo de condução. Já a "condução", consiste em realizar a seleção de trabalhos em fontes, seguindo os critérios primários e de qualidade também predefinidos, a fim de realizar a extração de dados daqueles selecionados. Após sintetizados, os dados são analisados de forma qualitativa e quantitativa com o intuito de responder às questões de pesquisa e fornecer informações 
consideradas relevantes para trabalhos futuros visando compor o "relato", última etapa da revisão. Esses processos são detalhados a seguir [KITCHENHAM, 2004].

\subsection{Planejamento}

A fase inicial da etapa de planejamento consiste em verificar a real necessidade de se desenvolver a revisão da literatura. Para isso, realizou-se uma busca inicial por revisões já publicadas e identificou-se que os trabalhos anteriores abordaram questões educacionais (cognitivas) [NETO et al. 2015], desenvolvimento centrado no usuário [OLIVEIRA et al. 2014] e quantitativos referentes a publicações [PIETRUCHINSKI et al. 2011]. Porém, os trabalhos citados não se preocuparam em pesquisar padrões entre as metodologias utilizadas no desenvolvimento dos jogos. Além disso, consideram um intervalo de tempo diferente do definido nessa revisão que engloba os últimos cinco anos.

Foi definido um protocolo para realização da revisão sistemática visando reduzir a possibilidade de manipulação por parte do pesquisador, pois este torna o processo mecânico e imparcial [KITCHENHAM 2004]. Nessa fase, define-se o escopo da pesquisa a fim de delimitar o objetivo a ser alcançado e as questões a serem respondidas, conforme Figura 1. Para isso, cada pergunta gera as palavras-chave necessárias para a localização de sua resposta. Essas palavras são, então, agrupadas formando uma única string de busca automática, que serve de ponto de partida para a localização de publicações.

\section{OBJETIVO}

\begin{tabular}{|c|}
\hline Revisar a literatura \\
científica a fim de \\
identificar as metodologias \\
utilizadas no \\
desenvolvimento de jogos \\
eletrônicos educacionais, no \\
contexto acadêmico, e os \\
requisitos metodológicos \\
específicos desse tipo de \\
processo, com ênfase na \\
documentação. \\
\hline
\end{tabular}

\section{STRING UTILIZADA NA BUSCA AUTOMÁTICA}

( ("serious game" OR "jogo sério" OR "educational game" OR "jogo educacional" ) AND ("game design") AND ("method" OR "metodologia" ) )

\section{Figura 1. Objetivo, questões de pesquisa do protocolo, com escolha preliminar das palavras chave sublinhadas e definição da string de busca automática.}

Considerando que, uma grande quantidade de palavras-chave e a definição de regras muito complexas e rígidas entre essas palavras restringem demasiadamente $o$ retorno da pesquisa, decidiu-se por identificar as melhores correspondências em língua inglesa, usar palavras compostas e excluir aquelas que poderiam ser buscadas na etapa de condução, aplicando os critérios de inclusão (CI) e de exclusão (CE).

Para inclusão, o artigo ou capítulo de livro (CI 4) deve ter sido publicado nos últimos cinco anos (CI 1), estar disponível em inglês ou português (CI 2), disponibilidade 
de acesso do texto completo (CI 3) e tratar especificamente do desenvolvimento de jogos eletrônicos no contexto educacional (CI 5).

Foram excluídos outros tipos de publicação (livros, resumos, relatórios técnicos, etc.) (CE 4), publicados a mais de cinco anos (CE 1), em uma língua diferente das especificadas (CE 2), com texto completo indisponível na base (CE 3), artigos repetidos (CE 6) e que não abordam o tema ou parte dele (CE 5) (apresentam apenas a aplicação de jogos (CE 5.1), utilizam jogos físicos (unplugged) (CE 5.2) ou não tem finalidade educacional (CE 5.3)). A língua inglesa foi utilizada por ser a mais comumente utilizada em artigos que abordam tecnologia e a portuguesa visa priorizar as publicações nacionais.

Como fonte de busca, utilizou-se a base geral Scopus para publicações internacionais e repositórios específicos locais relevantes ao tema de pesquisa, tais como: Simpósio Brasileiro de Informática na Educação (SBIE), o Workshop de Informática na Educação (WIE), a Revista Brasileira de Informática na Educação (RBIE) e a Revista Novas Tecnologias na Educação (Renote).

\subsection{Condução}

A etapa de condução, consiste em aplicar o protocolo de forma isenta e imparcial, a fim de obter um retorno objetivo e confiável. Dessa forma, ela tem início com a busca nas fontes predefinidas.

Após a aplicação da string nos mecanismos de busca automatizados, obteve-se um total de 356 textos. Foi possível aplicar, na própria busca automática, os critérios de exclusão números 1, 2 e 3, restando 55 textos após esse primeiro filtro. Em seguida, após uma leitura criteriosa das informações iniciais (título, resumo e palavras-chave) restaram 23 estudos primários. Os trabalhos foram excluídos pelo CE4 (12 trabalhos), CE 5.1 (10 trabalhos), CE 5.2 (2 trabalhos), CE 5.3 (7 trabalhos) e CE 6 (1 trabalho).

Como critério de qualidade (CQ), estabeleceu-se que o texto deveria abordar a metodologia utilizada, seja ela padronizada, híbrida ou autoral. Assim, após a leitura completa dos 29 trabalhos, 4 deles foram excluídos por indicar a metodologia em outro estudo, mantendo o foco apenas na aplicação ou no relato [SANTOS 2018] [HÄMÄLÄINEN et al. 2018], por realizar meta-análise [DESMET et al. 2016] ou entrevista semiestruturada [SLOOTMAKER et al. 2017] relacionada com gamificação. Dessa forma, restaram 19 trabalhos para extração de dados.

Para responder cada questão de pesquisa, estabeleceram-se dois dados específicos, apresentados na Figura 2, para extração durante a leitura completa, a fim de padronizar e classificar os trabalhos de acordo com os critérios da revisão. Os dados extraídos foram sintetizados e serviram de base para as análises e discussões.

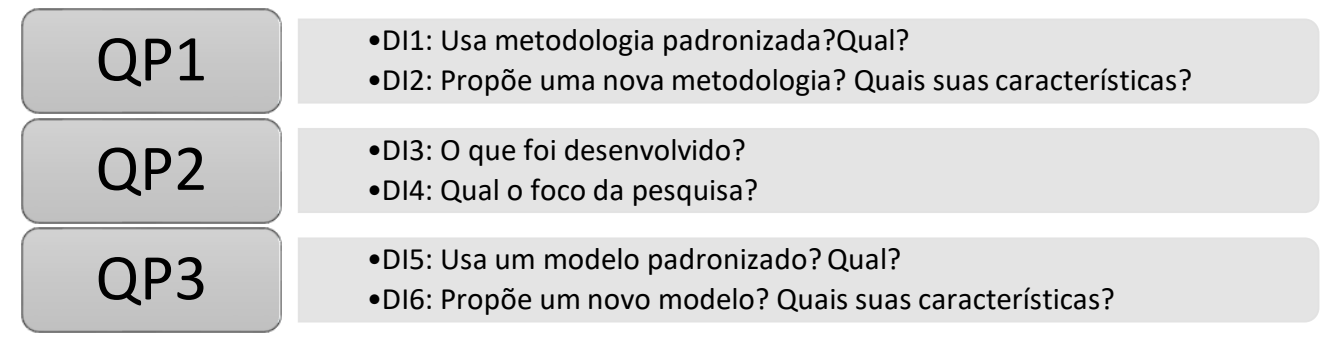

Figura 2. Data itens classificados por questão de pesquisa. 


\section{Síntese e discussão dos resultados}

A primeira questão de pesquisa visa identificar quais metodologias são utilizadas no desenvolvimento de jogos eletrônicos educacionais no contexto acadêmico. Nesse sentido houve a comprovação da hipótese inicial de que não há um processo padrão ou sequer uma referência metodológica, já que cada jogo é concebido a partir de uma necessidade, conteúdo e público específicos, vinculada a uma hipótese que, geralmente, parte de uma base pedagógica. Essa diversidade de possibilidades explica a diversidade de metodologias usadas e uma tendência a utilizar ferramentas ágeis ${ }^{1}$, mesmo que de forma não padronizada, e criação de protótipos (10 trabalhos).

\section{Gráfico 1. Metodologias de desenvolvimento utilizadas}
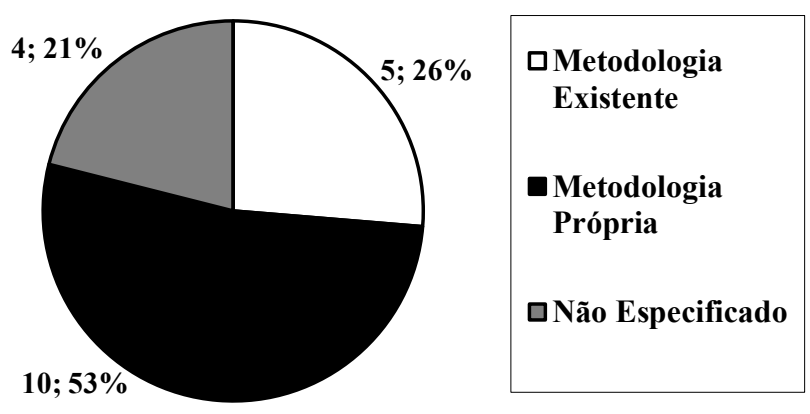

Como pode-se perceber, existe uma tendência em propor metodologias a partir de um problema específico, o que justifica o fato de uma mesma metodologia não ser citada em mais de um dos trabalhos analisados.

Chama a atenção o número expressivo de trabalhos que, apesar de se encaixarem nos critérios de inclusão e qualidade, não especificam uma metodologia, realizando apenas a descrição do processo de desenvolvimento e dos princípios norteadores. Um desses trabalhos cita, a "experiência do especialista" na descrição da metodologia utilizada, que representa uma tendência de simplificar ainda mais o processo, fazendo o uso de consultores com experiência no desenvolvimento de um tipo específico de jogo. Pode-se perceber, entre as metodologias padronizadas citadas, uma tendência de dividir o processo em etapas, com características típicas de metodologias ágeis, como prototipação, uso de iteratividade e pouca documentação.

A segunda questão de pesquisa buscou elencar requisitos metodológicos recorrentes no desenvolvimento de jogos educativos. Para responder a essa questão, procurou-se a especificação da prática realizada e o foco do estudo a fim de possibilitar um amplo entendimento da metodologia utilizada, independentemente de ser padronizada. Essa análise demonstrou a diversidade de meios usados para resolver problemas semelhantes, o que torna ainda mais complexa a tarefa de buscar padrões.

\footnotetext{
${ }^{1}$ Métodos Ágeis [AMBLER 2002], são mais flexíveis em relação aos tradicionais. Geralmente indicados para cenários onde há mudança constante de requisitos, resultados parciais devem ser entregues em pequenos espaços de tempo (iterações) e existe uma participação próxima do cliente com a equipe de desenvolvimento.
} 
No entanto, foi possível perceber algumas características comuns, principalmente no que se refere a objetivos metodológicos intrínsecos, oriundos das características específicas dos jogos eletrônicos educacionais, listadas a seguir.

a. Metodologias são adaptadas para cada caso específico: como o jogo educacional parte de uma necessidade específica de ensino, cada desenvolvedor avalia as ferramentas mais adequadas para o desenvolvimento de seu produto, sem usar um processo padronizado completo como base. Nesse sentido, há uma distinção entre metodologia de desenvolvimento e metodologia de documentação, pois cada etapa do processo pode usar uma metodologia própria;

b. Participação do usuário durante todo o processo de desenvolvimento: esse tópico é muito recorrente e prevê a presença do usuário (público alvo do processo de ensino) durante todo o processo de desenvolvimento, desde o levantamento de requisitos até os testes nos protótipos;

c. Relação entre teorias educacionais e o produto desenvolvido: surpreendentemente, poucos trabalhos citam diretamente a fundamentação por traz da mecânica do jogo, o que limita a comprovação de sua eficácia a aplicação do mesmo e a realização de testes;

d. Potencial para desenvolver o pensamento computacional: a participação do usuário no processo pode ser utilizada para o desenvolvimento de habilidades de pensamento computacional. Dessa forma, além do objetivo final do jogo, o processo tem potencial para apresentar a relação entre a mecânica e sua programação.

A terceira questão de pesquisa buscou saber como é feita a documentação do produto desenvolvido. Nesse sentido ficou comprovada a hipótese inicial que previa a despreocupação em documentar o jogo, uma vez que são poucos os trabalhos que se referem diretamente ao documento (3) de game design e, a maioria deles, apenas documenta, por meio de método próprio, as informações referentes a mecânica do jogo coletadas nas etapas iniciais de desenvolvimento, conforme Gráfico 2.

\section{Gráfico 2. Metodologias de documentação utilizadas}
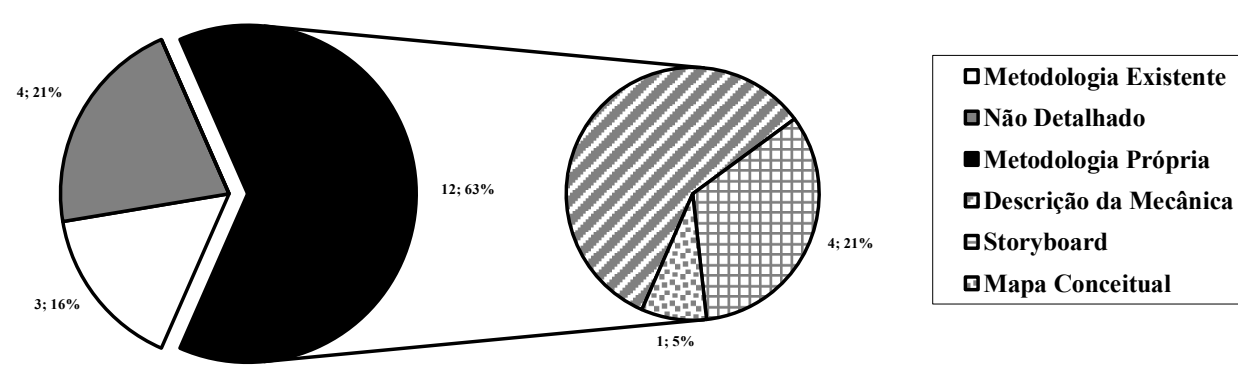

Importante destacar que a documentação utiliza características dos métodos ágeis sem citar diretamente essa metodologia, pois faz uso de uma variedade de ferramentas (diagramas, storyboards, mapas conceituais, tabelas, etc.) de acordo com a necessidade de representação das mecânicas do jogo (fases, personagens, ações, pontuação, etc.). 
IX Congresso Brasileiro de Informática na Educação (CBIE 2020)

Anais do XXXI Simpósio Brasileiro de Informática na Educação (SBIE 2020)

Outra técnica recorrente que remete a metodologias ágeis é o uso de iterações de prototipação. Muitos trabalhos realizam um breve levantamento de requisitos (geralmente sem uma referência direta a uma teoria educacional) e partem para a primeira iteração, que já tem como produto um protótipo da ideia. Uma fragilidade, no que se refere a essa documentação é que mesmo trabalhos que destacam a importância de documentação, não especificam a forma que pode ser feita e nenhum trabalho mencionou o controle de mudanças, o que infere que havendo alterações na ideia inicial elas não são incluídas na documentação do jogo.

Tabela 1. Síntese da extração de dados

\begin{tabular}{|c|c|c|c|c|c|c|c|}
\hline \multirow{2}{*}{ Artigo } & \multicolumn{2}{|r|}{ QP1 } & \multicolumn{2}{|r|}{ QP 2 } & \multicolumn{2}{|r|}{ QP 3} & \multirow{2}{*}{ PÚBLICO ALVO } \\
\hline & DI 1 & DI 2 & DI 3 & DI 4 & DI 5 & DI 6 & \\
\hline [ABIDIN et al. 2019] & Não utilizado & Não especificado & $\begin{array}{l}\text { Aplicação de } \\
\text { questionários }\end{array}$ & $\begin{array}{l}\text { Brain-based learning } \\
\text { Think aloud protocol }\end{array}$ & $\begin{array}{c}\text { Não } \\
\text { utilizado }\end{array}$ & $\begin{array}{c}\text { Storyboard } \\
\text { Protótipo de baixa } \\
\text { fidelidade }\end{array}$ & $\begin{array}{l}\text { ESTUDANTES COM } \\
\text { LEITURA LENTA }\end{array}$ \\
\hline [ALVES et al. 2019] & $\begin{array}{c}\text { [MOSER 2015] } \\
\text { Framework de 5 } \\
\text { etapas }\end{array}$ & $5 \begin{array}{c}\text { Framework de } 4 \\
\text { etapas }\end{array}$ & $\begin{array}{l}\text { Apresentação de } \\
\text { framework }\end{array}$ & \begin{tabular}{|c|} 
Design de jogos com \\
crianças \\
Conceitos de aprendizagem \\
e cognição
\end{tabular} & $\begin{array}{c}\text { Não } \\
\text { utilizado }\end{array}$ & Não detalhado & CRIANÇAS \\
\hline [ARAÚJO et al. 2016] & \begin{tabular}{|c|} 
SCHELL \\
$2008]$ \\
Game design de \\
4 etapas \\
\end{tabular} & Não especificado & $\begin{array}{c}\text { Oficinas de game } \\
\text { design para professores }\end{array}$ & \begin{tabular}{|c|} 
Game design como auxilio \\
na aprendizagem de \\
conteúdos diversos e de \\
computação.
\end{tabular} & $\begin{array}{c}\text { Não } \\
\text { utilizado }\end{array}$ & Não detalhado & NÃO ESPECIFICADO \\
\hline [BASTOS et al. 2017] & Não utilizado & Modelo em 3 etapas & Criação de jogo & Interação Natural (IN) & \begin{tabular}{|c|} 
Não \\
utilizado
\end{tabular} & $\begin{array}{c}\text { Descrição da } \\
\text { mecânica do jogo }\end{array}$ & CRIANÇAS \\
\hline [BUI et al. 2020] & Não utilizado & Não especificado & $\begin{array}{c}\text { Avaliação da } \\
\text { experiência do usuário }\end{array}$ & $\begin{array}{l}\text { Experiência de jogo } \\
\text { Design instrucional }\end{array}$ & $\begin{array}{c}\text { Não } \\
\text { utilizado }\end{array}$ & Não detalhado & $\begin{array}{l}\text { ESTUDANTES DE } \\
\text { ENSINO } \\
\text { FUNDAMENTAL } \\
\end{array}$ \\
\hline [CHEN et al. 2018] & Não utilizado & Tabela com 9 itens & Criação de Wiki & Uso de ferramentas on-line & \begin{tabular}{|c|} 
Não \\
utilizado \\
\end{tabular} & $\begin{array}{c}\text { Descrição da } \\
\text { mecânica do jogo }\end{array}$ & $\begin{array}{c}\text { ESTUDANTES DE PÓS } \\
\text { GRADUAÇÃO }\end{array}$ \\
\hline $\begin{array}{l}\text { [CZAUDERNA et al. } \\
\text { 2019] }\end{array}$ & Não utilizado & Não especificado & Criação de mini games & $\begin{array}{c}\text { Foco no jogador } \\
\text { Relação com objetivos } \\
\text { pedagógicos } \\
\text { Design instrucional } \\
\end{array}$ & $\begin{array}{c}\text { Não } \\
\text { utilizado }\end{array}$ & $\begin{array}{c}\text { Fluxogramas } \\
\text { storyboard } \\
\text { descrição da } \\
\text { mecânica do jogo } \\
\end{array}$ & CRIANÇAS \\
\hline $\begin{array}{l}\text { [EVANGELOPOULOU } \\
\text { et al. 2018] }\end{array}$ & $\begin{array}{l}\text { [IBRAHIM et } \\
\text { al. 2009] } \\
\text { Foca em } 3 \\
\text { dimensões } \\
\end{array}$ & Não especificado & Criação de jogo & $\begin{array}{c}\text { Objetivos educacionais } \\
\text { claros, diversão e feedback } \\
\text { imediato. }\end{array}$ & $\begin{array}{c}\text { Não } \\
\text { utilizado }\end{array}$ & $\begin{array}{l}\text { Descrição da } \\
\text { mecânica do jogo }\end{array}$ & NÃO ESPECIFICADO \\
\hline $\begin{array}{l}\text { [FERNANDES et al. } \\
\text { 2018] }\end{array}$ & Não utilizado & $\begin{array}{c}\text { Metodologia } \\
\text { dividida em } 4 \text { fases }\end{array}$ & $\begin{array}{c}\text { Criação de game } \\
\text { design usando a } \\
\text { metodologia thinking }\end{array}$ & $\begin{array}{l}\text { Motivação para aprender } \\
\text { pelos jogos colaborativos }\end{array}$ & $\begin{array}{c}\text { Não } \\
\text { utilizado }\end{array}$ & $\begin{array}{c}\text { Prevê templates } \\
\text { próprios para } \\
\text { descrição da } \\
\text { mecânica do jogo. }\end{array}$ & \begin{tabular}{|l} 
ESTUDANTES DE \\
GRADUAÇÃO E PÓS \\
GRADUAÇÃO
\end{tabular} \\
\hline [FLORES et al. 2020] & $\begin{array}{c}\text { [SCHWABER } \\
\text { 1995] } \\
\text { SCRUM }\end{array}$ & Não especificado & criação de jogo & $\begin{array}{c}\text { Vincula as Funções de } \\
\text { Aprendizagem e Ensino } \\
\text { (LTF) ao Design de Jogos } \\
\text { Padrões (PIB). }\end{array}$ & $\begin{array}{c}\text { Diagramas } \\
\text { UML }\end{array}$ & Não detalhado & $\begin{array}{l}\text { ESTUDANTES DE } \\
\text { GRADUAÇÃOO }\end{array}$ \\
\hline [HAFIS et al. 2018] & Não utilizado & $\begin{array}{c}\begin{array}{c}\text { Sistema bottom-up } \\
\text { de } 5 \text { etapas }\end{array} \\
\end{array}$ & $\begin{array}{l}\text { Teórico. Apresenta } \\
\text { requisitos de design }\end{array}$ & Trabalho colaborativo & \begin{tabular}{|c|} 
Não \\
utilizado \\
\end{tabular} & $\begin{array}{c}\text { Descrição da } \\
\text { mecânica do jogo }\end{array}$ & NÃO ESPECIFICADO \\
\hline [KAYALI et al. 2019] & Não utilizado & $\begin{array}{c}\text { Processo iterativo } \\
\text { de } 4 \text { etapas }\end{array}$ & $\begin{array}{l}\text { Curso de criação de } \\
\text { jogos }\end{array}$ & $\begin{array}{c}\text { Design thinking } \\
\text { Explorative design }\end{array}$ & \begin{tabular}{|c|} 
Não \\
utilizado \\
\end{tabular} & Blog & $\begin{array}{c}\text { ESTUDANTES DE } \\
\text { MESTRADO }\end{array}$ \\
\hline [MOITA et al. 2017] & Não utilizado & \begin{tabular}{|c|} 
Requisitos \\
Validação \\
Utilização de \\
metodologias ágeis \\
\end{tabular} & $\begin{array}{c}\text { criação de jogo usando } \\
\text { unity }\end{array}$ & \begin{tabular}{|c|} 
Compreensão e interação do \\
usuário \\
Pressupostos da IHC e do \\
design de games. \\
\end{tabular} & $\begin{array}{c}\text { Diagramas } \\
\text { UML }\end{array}$ & Não detalhado & AUTISTAS \\
\hline [ROCHA et al. 2015] & {$\left[\begin{array}{c}\text { [ROCHA 2014] } \\
\text { Metodologia de } \\
7 \text { etapas }\end{array}\right.$} & $\begin{array}{c}\text { NÃO } \\
\text { ESPECIFICADO }\end{array}$ & $\begin{array}{l}\text { Teórico. Apresenta } \\
\text { metodologia. }\end{array}$ & $\begin{array}{c}\text { Interação } \\
\text { integração } \\
\text { Generalização } \\
\text { Uso de ferramentas livres } \\
\text { Design instrucional } \\
\end{array}$ & $\begin{array}{c}\text { Diagramas } \\
\text { UML }\end{array}$ & Storyboard & NÃO ESPECIFICADO \\
\hline $\begin{array}{c}\text { [ROSMANSYAH et al. } \\
2019]\end{array}$ & $\begin{array}{c}\text { [ALDOOBIE } \\
\text { 2015] } \\
\begin{array}{c}\text { Metdologia de 5 } \\
\text { etapas }\end{array} \\
\end{array}$ & $\begin{array}{c}\text { NÃO } \\
\text { ESPECIFICADO }\end{array}$ & $\begin{array}{l}\text { Planejamento e } \\
\text { simulação }\end{array}$ & Design instrucional & $\begin{array}{c}\text { Não } \\
\text { utilizado }\end{array}$ & $\begin{array}{l}\text { Descrição da } \\
\text { mecânica do jogo }\end{array}$ & NÃO ESPECIFICADO \\
\hline [ROUNGAS et al. 2019] & Não utilizado & $\begin{array}{c}\text { Framework com } 9 \\
\text { etapas }\end{array}$ & $\begin{array}{l}\text { Teórico. Apresenta } \\
\text { framework. }\end{array}$ & Tomada de decisão & \begin{tabular}{|c|} 
Não \\
utilizado \\
\end{tabular} & Mapas conceituais & NÃO ESPECIFICADO \\
\hline $\begin{array}{l}\text { [SANTANA-MANCILLA } \\
\text { et al. 2019] }\end{array}$ & Não utilizado & $\begin{array}{c}\begin{array}{c}\text { Definição de } 3 \\
\text { elementos }\end{array} \\
\end{array}$ & Criação de 2 jogos & Participação do usuário & \begin{tabular}{|c|} 
Não \\
utilizado \\
\end{tabular} & $\begin{array}{c}\text { Descrição da } \\
\text { mecânica do jogo }\end{array}$ & $\begin{array}{l}\text { ESTUDANTES DE } \\
\text { GRADUAÇÃOO }\end{array}$ \\
\hline [SCHULTZ et al. 2018] & Não utilizado & $\begin{array}{c}\text { "experiência do } \\
\text { especialista" }\end{array}$ & $\begin{array}{c}\text { Oficinas de } \\
\text { desenvolvimento }\end{array}$ & Aprendizagem ativa & \begin{tabular}{|c|} 
Não \\
utilizado \\
\end{tabular} & Storyboard & $\begin{array}{c}\text { PESSOAS } \\
\text { VULNERÁVEIS } \\
\end{array}$ \\
\hline [ZHENG 2019] & Não utilizado & $\begin{array}{c}\text { Processo em } 6 \\
\text { etapas }\end{array}$ & $\begin{array}{l}\text { Curso de design de } \\
\text { jogos }\end{array}$ & $\begin{array}{c}\text { Teoria construtivista } \\
\text { Múltiplas inteligências } \\
\text { Aprendizado de jogos } \\
\text { Desenvolvimento da } \\
\text { autonomia } \\
\end{array}$ & $\begin{array}{c}\text { Não } \\
\text { utilizado }\end{array}$ & Não detalhado & NÃO ESPECIFICADO \\
\hline
\end{tabular}

Percebe- se que, existem boas práticas relacionando padrões de design de jogos a públicos específicos com processos diversos de desenvolvimento e documentação (Tabela 1). No entanto, não se identificou, nos trabalhos analisados, uma metodologia para apoiar o desenvolvimento em um contexto geral. Fica claro que a diversidade de técnicas existentes na literatura (mais ou menos formais) representa a adaptação 
necessária para projetar cada produto a partir de uma necessidade de ensino para cada público específico, o que justifica a inclinação ao uso de técnicas ágeis de desenvolvimento e à simplificação da documentação.

\section{Considerações Finais}

Essa revisão se propôs a mapear similaridades em metodologias utilizadas na produção de jogos Educativos. Tal tarefa se mostrou desafiadora à medida que se percebeu que as metodologias são adaptadas para cada tipo de produto, não havendo padronização de ferramentas e técnicas de desenvolvimento. Cada processo é único e de adapta ao público e ao problema, sempre devendo ser relacionado a seu objetivo pedagógico.

Além disso, após a extração de dados, foi possível identificar padrões, que foram apresentados como boas práticas a fim de apontar potencialidades e fragilidades em projetos de desenvolvimento de jogos educativos. A participação dos usuários no processo de desenvolvimento foi a característica mais recorrente, uma vez que grande parte dos projetos intencionava o ensino concomitante da computação. No entanto, ter clareza nos objetivos de aprendizado, pode ser considerada uma fragilidade, pois raramente são considerados ao longo do design, e tampouco associados à mecânica do jogo. Nesse sentido, muitas abordagens recomendam uma relação entre design de jogos e design instrucional.

Apesar de não haver uma metodologia padrão, cada autor busca práticas nas metodologias de desenvolvimento existentes que vão ao encontro de fatores relacionados ao processo de ensino-aprendizagem a fim de criar um modelo eficiente para um caso específico, o que cria uma grande diversidade de novas metodologias. Dessa forma, seria importante desenvolver uma abordagem para definição de processos afim de propor uma metodologia dinâmica e adaptativa que apontasse critérios importantes de design para cada tipo de desenvolvimento de jogos eletrônicos educacionais.

\section{Referências}

Abidin, S. R. Z. Noor, S. F. M. Ashaari, N, S. (2019) "Low-fidelity Prototype Design for Serious Game for Slow-reading Students", International Journal of Advanced Computer Science and Applications, Vol. 10, No. 3.

Aldoobie, N. (2015) “ADDIE Model”. America International Journal of Contemporary Research, 5(6), 68-72.

Ambler, S. (2002) “Agile modeling”. New York: Wiley Computer Publishing.

Araújo, G. G. Silva, T. R. Aranha, E. (2016) “A construção de jogos digitais na escola: um relato de experiência na formação de professores", XXII Workshop de Informática na Escola.

Alves, A. G. Hotins, R. C. L. Raabe, A. L. A. (2019) ““"Eu fiz meu game”: um framework para desenvolvimento de jogos por crianças", Revista Brasileira de Informática na Educação - RBIE, 27(2), 218-238.

Bastos, M. L. Marques, P. C. F. Dantas, M. W. G. Silva, L. N. Silva, J. S. (2017) “Jogo Conhecendo o Espaço do Museu de Oceanografia: utilizando interface natural no processo de ensino aprendizagem", XXVIII Simpósio Brasileiro de Informática na Educação. 
Bui, P., Rodríguez-Aflecht, G., Brezovszky, B. et al. (2020) “Understanding students' game experiences throughout the developmental process of the number navigation game", Education Tech Research Dev. https://doi.org/10.1007/s11423-020-09755-8.

Chen, C. Huang, C. Gribbins, M. Swan, K. (2018) “Gamify Online Courses With Tools Built Into Your Learning Management System (LMS) to Enhance Self-Determined and Active Learning”, Online Learning Journal, Volume 22 Issue 3.

Czauderna, A. Guardiola, E. (2019) "The Gameplay Loop Methodology as a Tool for Educational Game Design” Electronic Journal of e-Learning, 17(3), pp. 207-221.

DeSmet, A. Thompson, D. Baranowski, D. Palmeira, A. Verloigne, M. De Bourdeaudhuij, I. (2016) "Is Participatory Design Associated with the Effectiveness of Serious Digital Games for Healthy Lifestyle Promotion? A Meta-Analysis”, Journal of Medical Internet Research, vol. 18, iss. 4.

Evangelopoulou, O. Xinogalos, S. (2018) "Myth Troubles: An Open-Source Educational Game in Scratch for Greek Mythology", Simulation and Gaming, Vol. 49(1) 72-91.

Fernandes, K. T. Lucena, M. J. N. R. Aranha, E. H. S. (2018) "Uma Experiência na Criação de Game Design de Jogos Digitais Educativos a partir do Design Thinking”, Revista Novas Tecnologias na Educação - Renote. V. 16 N$^{\circ} 1$.

Flores, N. Paiva, A. C. R. Cruz, N. (2020) "Teaching Software Engineering Topics Through Pedagogical Game Design Patterns: An Empirical Study”, Information, 11, 53.

Hafis, M. Supianto, A. A. (2018) "Mobile Game Design for Learning Chemical Bonds with Endless Run Approach", International Journal of Interactive Mobile Technologies, Vol 12, No 8, 104-112.

Hämäläinen, R. H. Niilo-Rämä, M. Lainema, T. Oksanen, K. (2018) "How to Raise Different Game Collaboration Activities: The Association Between Game Mechanics, Players' Roles and Collaboration Processes", Simulation and Gaming, Vol. 49(1) 5071.

Ibrahim, R. Jaafar, A. (2009) "Educational games (EG) design framework: Combination of game design, pedagogy and content modeling". International Conference on Electrical Engineering and Informatics, 2009. ICEEI'09 (Vol. 1, pp. 293-298). IEEE. doi:10.1109/ ICEEI.2009.5254771

Kayali, F. Luckner, N. Purgathofer, P. (2019) “Teaching Gameful Design”, ACM International Conference Proceeding Series, https://doi.org/10.1145/3337722. 3341823, August.

Kitchenham, B. (2004) "Procedures for Performing Systematic Reviews", Technical Report TR/SE-0401. Department of Computer Science, Keele University and National ICT. Australia.

Moita, F. M. G. S. C. Viana, L. H. Medeiros, F. M. Candido, V. M. A. (2017) "Design e desenvolvimento de um game assistivo para autistas", XXVIII Simpósio Brasileiro de Informática na Educação.

Moser, C. (2015). “Child-Centered Game Development”. Salzburg, Áustria. 
Neto, J. C. Reinehr, S. Malucelli, A. (2015) "Processo de Desenvolvimento para Jogos Eletrônicos Educacionais: uma Revisão de Literatura", Revista Brasileira de Informática na Educação, Volume 23, Número 2.

Oliveira, H. C. Hounsell, M. S. Kemczinski, A. (2014) "Mapeamento sistemático de metodologias de desenvolvimento centrado no usuário para jogos sérios", Simpósio Brasileiro de Informática na Educação.

Perucia, A. Berthêm, A. Bertschinger, G. Castro, R. R. (2007) “Desenvolvimento de jogos eletrônicos: teoria e prática”. 2. ed. Novatec, São Paulo.

Pietruchinski, M. H. Neto, J. C. Malucelli, A. Reinehr, S. (2011) "Os jogos educativos no contexto do SBIE: uma revisão sistemática de Literatura". Simpósio Brasileiro de Informática na Educação.

Rocha, R. V. (2014) "Metodologia iterativa e modelos integradores para desenvolvimento de jogos sérios de treinamento e avaliação de desempenho humano". Tese (Doutorado em Ciência da Computação) - Departamento de Computação, Universidade de São Carlos, São Carlos, SP.

Rocha, R. V. Zem-Lopes, A. M. Pedro, L. Z. Bittencourt, I. I. Isotani, S. (2015) "Metodologia de Desenvolvimento de Jogos Sérios: especificação de ferramentas de apoio open source”, XXVI Simpósio Brasileiro de Informática na Educação.

Rosmansyah, Y. Achiruzaman, M. Hardi, A. B. (2019) “A 3d Multiuser Virtual Learning Environment for Online Training of Agriculture Surveyors", Journal of Information Technology Education: Research, Volume 18, 481-507.

Roungas, B. Bekius, F. Meijer, S. (2019) "The Game Between Game Theory and Gaming Simulations: Design Choices", Simulation and Gaming, Vol. 50(2) 180-201.

Santana-Mancilla, P. C. Rodriguez-Ortiz, M. A. Garcia-Ruiz, M. A. Gaytan-Lugo, L. S. Fajardo-Flores, S. B. Contreras-Castillo, J. (2019) "Teaching HCI Skills in Higher Education through Game Design: A Study of Students' Perceptions”, Informatics, 6(2), 22.

Santos, W. O. (2018) "EGV: A Methodology Proposal to Educational Games Virtualization", Revista Novas Tecnologias na Educação - Renote. V. 16 N $^{\circ} 1$.

Schell, J. (2008) "The Art of Game design: A Book of Lenses". Burlington: Elsevier.

Schwaber, K. (1995) "Scrum Development Process". OOPSLA Business Object Design and Implementation Workshop.

Schultz, E. P. B. Ortiz, J. S. B. García, L. S. Pereira, R. (2018) “Teaching Game Design and Basic Computing Concepts: a democratic experiment in a Socioeconomically Vulnerable Community”, XXIX Simpósio Brasileiro de Informática na Educação.

Slootmaker, A. Hummel, H. Koper, R. (2017) "Evaluating the Usability of Authoring Environments for Serious Games", Simulation and Gaming, Vol. 48(4) 553-578.

Zheng. Y. (2019) "3D Course Teaching Based on Educational Game Development Theory - Case Study of Game Design Course", International Journal of Emerging Technologies in Learning, Vol 14, No 02, 54-68. 